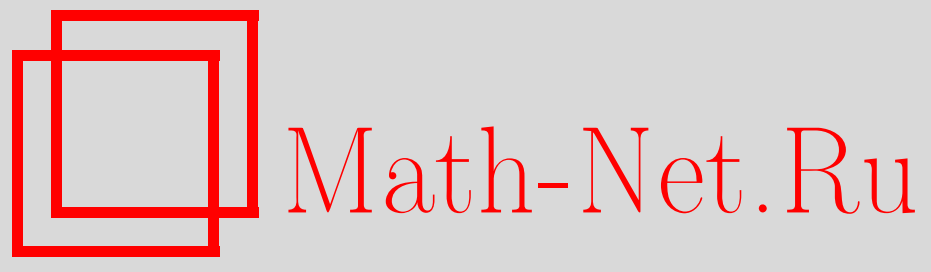

Б. Г. Вакулов, Операторы сферической свертки в пространствах переменной гёльдеровости, Матем. заметки, 2006, том 80, выпуск 5, 683-695

DOI: https://doi.org/10.4213/mzm3077

Использование Общероссийского математического портала Math-Net.Ru подразумевает, что вы прочитали и согласны с пользовательским соглашением http://www . mathnet.ru/rus/agreement

Параметры загрузки:

IP : 54.157 .27 .8

26 апреля 2023 г., 18:13:45

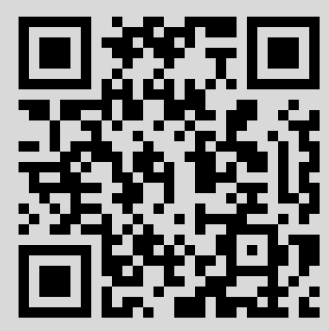




\section{ОПЕРАТОРЫ СФЕРИЧЕСКОЙ СВЕРТКИ В ПРОСТРАНСТВАХ ПЕРЕМЕННОЙ ГЁЛЬДЕРОВОСТИ}

\section{Б. Г. Вакулов}

В работе исследуются образы оператора типа сферического потенциала комплексного порядка и сферических сверток с ядрами, зависящими от скалярного произведения и имеющими мультипликатор по сферическим гармоникам заданной асимптотики на бесконечности. На основании теорем о действии этих операторов в пространствах переменной гёльдеровости строятся изоморфизмы этих пространств. Изучается действие в пространствах Гёльдера переменного порядка сферических потенциалов с особенностями на полюсах сферы. С помощью стереографической проекции получаются аналогичные изоморфизмы пространств переменной гёльдеровости по $n$-мерному евклидову пространству (в случае его одноточечной компактификации) с некоторыми степенными весами.

Библиография: 22 названия.

Введение. В работе исследуются сферический оператор типа потенциала $K^{\alpha}$ и связанный с ним гиперсингулярный интеграл $D^{\alpha}$ в случае комплексных порядков. Оператор типа потенциала $K^{\alpha} f$ при $\operatorname{Re} \alpha>0$ определяется как оператор сферической свертки в виде

$$
\left(K^{\alpha} f\right)(x)=\frac{1}{\gamma_{n-1}(\alpha)} \int_{S_{n-1}} \frac{f(\sigma)}{|x-\sigma|^{n-1-\alpha}} d \sigma, \quad x \in S_{n-1},
$$

где $\gamma_{n-1}(\alpha)$ - нормировочная константа (см. вспомогательные сведения в п. 1) и $\alpha \neq n-1, n+1, \ldots$. При $\alpha=n-1, n+1, \ldots$ оператор $K^{\alpha}$ модифицируется обычным образом (см., например, [1]-[4]). Для $0 \leqslant \operatorname{Re} \alpha<2$ сферический гиперсингулярный интеграл $D^{\alpha} f$ определяется следующим образом:

$$
\left(D^{\alpha} f\right)(x)=\frac{1}{\gamma_{n-1}(-\alpha)} \lim _{\varepsilon \rightarrow 0} \int_{\substack{S_{n-1} \\|x-\sigma| \geqslant \varepsilon}} \frac{f(\sigma)-f(x)}{|x-\sigma|^{n-1+\alpha}} d \sigma, \quad x \in S_{n-1} .
$$

В работах [5]-[11] для сферического потенциала $K^{\alpha}$, гиперсингулярного интеграла $D^{\alpha}$ при $\operatorname{Re} \alpha \neq 0$ и $D^{i \theta}\left(\forall \theta \in \mathbb{R}^{1} \backslash\{0\}\right)$ при $\operatorname{Re} \alpha=0$ выясняется их действие в обобщенных классах Гёльдера $H^{\omega}\left(S_{n-1}\right)$. Там предполагалось, что характеристика $\omega(t)$ принадлежит классу $\Phi_{\beta}^{\delta}$ типа Бари-Стечкина, причем $\beta$ и $\delta$ определяются в зависимости от $n$ и $\operatorname{Re} \alpha$. Наряду с операторами $K^{\alpha}, D^{\alpha}$ в [8]-[10], [12] рассматривались также операторы

$$
\mathscr{D}^{\alpha}=\frac{1}{b_{n}} I+D^{\alpha}, \quad b_{n}=\frac{\Gamma((n-1-\alpha) / 2)}{\Gamma((n-1+\alpha) / 2)},
$$

(C) Б. Г. ВАкулов, 2006 
для которых при $0 \leqslant \operatorname{Re} \alpha<2, \alpha \neq 0$, справедливы соотношения

$$
\mathscr{D}^{\alpha} K^{\alpha}=I, \quad K^{\alpha} \mathscr{D}^{\alpha}=I, \quad 0<\operatorname{Re} \alpha<2, \quad \mathscr{D}^{i \theta} \mathscr{D}^{-i \theta}=I \quad \forall \theta \in \mathbb{R}^{1} \backslash\{0\} .
$$

На основе полученных результатов, в частности, при $\omega(t)=t^{\lambda}$, показывалось, что оператор $K^{\alpha}$ с $0 \leqslant \operatorname{Re} \alpha<1$ осуществляет следующий изоморфизм:

$$
\begin{gathered}
K^{\alpha}\left[H^{\lambda}\left(S_{n-1}\right)\right]=H^{\lambda+\operatorname{Re} \alpha}\left(S_{n-1}\right), \quad 0<\lambda, \quad \operatorname{Re} \alpha<1, \quad \lambda+\operatorname{Re} \alpha<1, \\
\mathscr{D}^{ \pm i \theta}\left[H^{\lambda}\left(S_{n-1}\right)\right]=H^{\lambda}\left(S_{n-1}\right) \quad \forall \theta \in \mathbb{R}^{1} \backslash\{0\}, \quad 0<\lambda<1 .
\end{gathered}
$$

В [7]-[10] эти результаты обобщались на случай степенного веса.

В настоящей работе операторы $(0.1)-(0.3)$ изучаются в пространствах переменной гёльдеровости. Центральным результатом здесь является получение изоморфизмов

$$
\begin{gathered}
K^{\alpha}\left[H_{k}^{\lambda(x)}\left(S_{n-1}\right)\right]=H_{k+[\operatorname{Re} \alpha]}^{\lambda(x)+\{\operatorname{Re} \alpha\}}\left(S_{n-1}\right), \\
\mathscr{D}^{ \pm i \theta}\left[H_{k}^{\lambda(x)}\left(S_{n-1}\right)\right]=H_{k}^{\lambda(x)}\left(S_{n-1}\right) \quad \forall \theta \in \mathbb{R}^{1} \backslash\{0\},
\end{gathered}
$$

при условиях

$$
\operatorname{Re} \alpha>0, \quad \inf _{x \in S_{n-1}} \lambda(x)>0, \quad\{\operatorname{Re} \alpha\}+\sup _{x \in S_{n-1}} \lambda(x)<1
$$

и в предположении, что $\lambda(x)$ удовлетворяет условию

$$
|\lambda(y)-\lambda(x)| \leqslant c|\ln | x-y||^{-1}, \quad x, y \in S_{n-1}, \quad|x-y| \leqslant \frac{1}{2} .
$$

Для $K^{\alpha}$ рассмотрен также случай $\operatorname{Re} \alpha+\sup _{x \in S_{n-1}} \lambda(x)=1$.

В качестве следствия получен также (с помощью стереографической проекции) изоморфизм пространств переменной гёльдеровости с некоторыми степенными весами в $\mathbb{R}^{n}$, осуществляемый риссовым потенциалом по $\mathbb{R}^{n}$.

Отметим еще, что дробные интегралы и дробные производные на отрезке вещественной оси в пространствах Гёльдера переменного порядка ранее рассматривались в [11], [13]-[15]. При изучении дробной производной, а следовательно, и при установлении соответствующего изоморфизма и там возникало условие типа (0.9).

Все встречающиеся ниже постоянные, если не оговорено, будем обозначать одной $c$. Кроме того, считаем $n \geqslant 3$. Случай $n=2$ несколько отличается технически, но также может быть рассмотрен.

1. Вспомогательные сведения. Будем использовать следующие стандартные обозначения многомерного анализа на сфере $S_{n-1}$ в $\mathbb{R}^{n}$ :

$$
\begin{gathered}
x=\left(x_{1}, x_{2}, \ldots, x_{n}\right), \quad|x|=\sqrt{x_{1}^{2}+x_{2}^{2}+\cdots+x_{n}^{2}}, \quad x \cdot y=x_{1} y_{1}+x_{2} y_{2}+\cdots+x_{n} y_{n}, \\
S_{n-1}=\left\{x: x \in \mathbb{R}^{n},|x|=1\right\}, \quad\left|S_{n-1}\right|=2 \pi^{(n-1) / 2} \Gamma^{-1}\left(\frac{n-1}{2}\right), \\
\gamma_{n-1}(\alpha)=2^{\alpha} \pi^{(n-1) / 2} \Gamma\left(\frac{\alpha}{2}\right) \Gamma^{-1}\left(\frac{n-1-\alpha}{2}\right) .
\end{gathered}
$$

Отметим, что для придания смысла нормировочному коэффициенту $\gamma_{n-1}(\alpha)$ при $\operatorname{Re} \alpha \leqslant 0, \alpha \neq 0,-2 \ldots$ следует в его выражении множитель $\Gamma(\alpha / 2)$ понимать как аналитическое продолжение по $\alpha$. 
Приведем некоторые факты, которыми будем пользоваться. Известен следующий частный случай формулы Функа-Гекке, называемый также формулой Каталана (см., например, [16; с. 20]):

$$
\int_{S_{n-1}} f(x \cdot \sigma) d \sigma=C_{n} \int_{-1}^{1} f(t)\left(1-t^{2}\right)^{(n-3) / 2} d t, \quad x \in S_{n-1},
$$

где $C_{n}=\left|S_{n-2}\right|=2 \pi^{(n-1) / 2} \Gamma^{-1}((n-1) / 2)$ означает площадь единичной сферы в $\mathbb{R}^{n-1}$. С помощью метода вращений, который используется для доказательства формулы (1.1), и с учетом того, что $|x-\sigma|=\sqrt{2(1-x \cdot \sigma)}$ вычисляются и оцениваются интегралы вида

$$
J(a, b, x)=\int_{a<|x-\sigma|<b} g(|x-\sigma|, x) d \sigma, \quad x \in S_{n-1},
$$

где $0 \leqslant a<b \leqslant 2$. А именно, справедлива

ЛЕмма 1.1. Пусть $n \geqslant 2$. Тогда с учетом (1.2) имеем

$$
J(a, b, x)=2^{3-n} C_{n} \int_{a}^{b} g(u, x) u^{n-2}\left(4-u^{2}\right)^{(n-3) / 2} d u,
$$

u, в частности, при $n \geqslant 3$

$$
|J(a, b, x)| \leqslant C_{n} \int_{a}^{b}|g(u, x)| u^{n-2} d u .
$$

Из формулы (1.3), в частности, следует, что

$$
\int_{S_{n-1}} \frac{d \sigma}{|x-\sigma|^{n-1-\alpha}}=\gamma_{n-1}(\alpha), \quad \operatorname{Re} \alpha>0 .
$$

Отметим еще, что

$$
\int_{a<|x-\sigma|<b} g(|x-\sigma|) d \sigma=\int_{a<|y-\sigma|<b} g(|y-\sigma|) d \sigma \quad \forall x, y \in S_{n-1} .
$$

Следующая оценка, представляющая интерес при $\gamma>n-1$, выводится из формулы (1.4):

$$
\int_{|y-\sigma|>2|x-y|} \frac{d \sigma}{|\sigma-y|^{\gamma}} \leqslant \int_{|y-\sigma|>|x-y|} \frac{d \sigma}{|\sigma-y|^{\gamma}} \leqslant c|x-y|^{-\gamma+n-1}, \quad \gamma>n-1,
$$

см. подробности в [3], [7].

В дальнейшем нам понадобятся известные классические числовые неравенства, которые мы приведем здесь для случая комплексных показателей:

$$
\begin{array}{ll}
\left|x^{\mu}-y^{\mu}\right| \leqslant c|x-y| x^{\operatorname{Re} \mu-1}, & x \geqslant y>0, \quad \operatorname{Re} \mu \geqslant 0, \\
\left|x^{\mu}-y^{\mu}\right| \leqslant c|x-y|^{\operatorname{Re} \mu-1}, & x \geqslant y>0, \quad \operatorname{Re} \mu \leqslant 1, \\
\left|x^{\mu}-y^{\mu}\right| \leqslant c|x-y|^{\operatorname{Re} \mu}, & x>0, \quad y>0, \quad 0<\operatorname{Re} \mu \leqslant 1, \\
\left|x^{\mu}-y^{\mu}\right| \leqslant c|x-y|(x+y)^{\operatorname{Re} \mu-1}, & x>0, \quad y>0, \quad \operatorname{Re} \mu \geqslant 1 .
\end{array}
$$

При оценке сферических операторов возникает необходимость в оценках на части сферы, определяемой неравенством $|x-\sigma| \geqslant 2|x-y|$. 
ЛЕмма 1.2. Пусть $x, y \in S_{n-1} u$

$$
|x-\sigma| \geqslant 2|x-y| \text {. }
$$

Тогда при $\operatorname{Re} \gamma>0$ справедливы неравенства

$$
|| x-\left.\sigma\right|^{-\gamma}-|y-\sigma|^{-\gamma} \mid \leqslant c \frac{|x-y|}{|x-\sigma|^{\operatorname{Re} \gamma}(|x-\sigma|+|x-y|)} \leqslant c \frac{|x-y|}{|x-\sigma|^{\operatorname{Re} \gamma+1}} .
$$

Для доказательства леммы 1.2 вначале заметим, что из упомянутых выше числовых неравенств (1.7)-(1.10) выводится следующее: пусть $a>0, b>0$ и, кроме того, $a \geqslant 2|b-a|, \operatorname{Re} \gamma>0$; тогда справедливо неравенство

$$
\left|a^{-\gamma}-b^{-\gamma}\right| \leqslant \frac{c}{a^{\operatorname{Re} \gamma}} \cdot \frac{|a-b|}{a+|a-b|} .
$$

Чтобы вывести отсюда лемму 1.2, полагаем $a=|x-\sigma|, b=|y-\sigma|$, так что условие $a \geqslant 2|b-a|$ выполнено в силу (1.11). Остается применить (1.13) и воспользоваться монотонным возрастанием по $\xi$ функции $\xi /(a+\xi), a>0, \xi>0$, что приводит к (1.12), см. подробности в [7].

ОПРЕДЕЛЕНИЕ 1.1. Через $H^{\lambda(x)}\left(S_{n-1}\right)$ обозначим банахово пространство непрерывных на $S_{n-1}$ функций, для которых конечна норма

$$
\|f\|_{\lambda}=\|f\|_{C}+A_{f}, \quad A_{f}=\sup _{x, y \in S_{n-1}} \frac{|f(x)-f(y)|}{|x-y|^{\lambda(x)}}=\sup _{x, y \in S_{n-1}} \frac{|f(x)-f(y)|}{|x-y|^{\lambda(y)}} .
$$

Всюду в дальнейшем обозначаем

$$
\lambda_{-}=\inf _{x \in S_{n-1}} \lambda(x), \quad \lambda_{+}=\sup _{x \in S_{n-1}} \lambda(x),
$$

и предполагаем, что

$$
0<\lambda_{-} \leqslant \lambda(x) \leqslant \lambda_{+}<1 .
$$

Через $K$ обозначаем оператор сферической свертки, инвариантный относительно всех вращений на сфере,

$$
(K f)(x)=\int_{S_{n-1}} k(x \cdot \sigma) f(\sigma) d \sigma, \quad x \in S_{n-1} .
$$

Пусть $f_{m \nu}$ - коэффициенты $f(x) \in C^{\infty}\left(S_{n-1}\right)$ при ее разложении в ряд ФурьеЛапласа по сферическим гармоникам; как известно, действие оператора $K$ на сферических гармониках сводится к умножению $f_{m \nu}$ на последовательность $\left\{k_{m}\right\}_{m=0}^{\infty}$, называемую мультипликатором Фурье-Лапласа оператора $K$ и определяемую через ядро $k(t),-1<t<1$, по формуле

$$
k_{m}=\left|S_{n-2}\right| \int_{-1}^{1} k(t) P_{m}(t)\left(1-t^{2}\right)^{(n-2) / 2} d t,
$$

где $P_{m}(t)=\left(C_{m+n-3}^{m}\right)^{-1} C_{m}^{(n-2) / 2}(t)$ и $C_{m}^{(n-2) / 2}(t)$ - многочлены Гегенбауэра. 
ОПРЕДЕЛЕНИЕ 1.2. Через $W_{\lambda, N}$ обозначим класс мультипликаторов $\left\{k_{m}\right\}_{m=0}^{\infty}$ по сферическим гармоникам, $\left|k_{m}\right|<\infty$, допускающих асимптотику вида

$$
k_{m}=\sum_{j=0}^{N} c_{j} m^{\lambda-j}+O\left(m^{\lambda-N-\varepsilon}\right), \quad m \rightarrow \infty,
$$

где $c_{0} \neq 0, \varepsilon>0, \lambda \in \mathbb{C}^{1}, N=0,1, \ldots$.

Еще нам понадобятся следующие две леммы.

Лемма 1.3. Если $\left\{k_{m}\right\}_{m=0}^{\infty} \in W_{\lambda, N} u k_{m} \neq 0, m=0,1, \ldots, m o\left\{k_{m}^{-1}\right\}_{m=0}^{\infty} \in W_{\lambda, N}$. Eсли $\left\{k_{m}\right\}_{m=0}^{\infty} \in W_{\lambda, N},\left\{l_{m}\right\}_{m=0}^{\infty} \in W_{\mu, N}, \operatorname{mo}\left\{k_{m} l_{m}\right\}_{m=0}^{\infty} \in W_{\lambda+\mu, N}$.

Доказывается непосредственной проверкой.

Лемма 1.4. Если $\left\{k_{m}\right\}_{m=0}^{\infty} \in W_{-\lambda, N}$, где $\operatorname{Re} \lambda>0, \lambda \neq n-1+2 k, k=0,1,2, \ldots$, $N \geqslant[(n+1) / 2]$, то оператор, отвечающий этому мультипликатору, имеет вид

$$
A \varphi=K^{\lambda}(a I+B) \varphi=K^{\lambda}\left(a \varphi+\int_{S_{n-1}} k(x \cdot \sigma) \varphi(\sigma) d \sigma\right),
$$

где $k(t) \in L_{1}\left([-1,1],\left(1-t^{2}\right)^{(n-3) / 2}\right)$ u $a=\lim _{m \rightarrow \infty} m^{\lambda} k_{m}$. Eсли $N>n+1-\operatorname{Re} \lambda$, mo оператор, отвечающий этому мультипликатору, имеет вид

$$
A \varphi=\int_{S_{n-1}} k(x \cdot \sigma) \varphi(\sigma) d \sigma+\sum_{j=0}^{N} c_{j} K^{\lambda+j}
$$

в случае $\operatorname{Re} \lambda>0 u$

$$
A \varphi=c_{0} \varphi+\int_{S_{n-1}} k(x \cdot \sigma) \varphi(\sigma) d \sigma+\sum_{j=1}^{N} c_{j} K^{j}
$$

в случае $\lambda=0$. Здесъ $k(t) \in C^{1}([-1,1]), c_{0}=\lim _{m \rightarrow \infty} m^{\lambda} k_{m}$.

Лемма 1.4 следует из вида мультипликатора по сферическим гармоникам для оператора (0.1):

$$
k_{m}=\frac{\Gamma(m+(n-1-\alpha) / 2)}{\Gamma(m+(n-1+\alpha) / 2)},
$$

известного асимптотического представления для $\Gamma(z+a) / \Gamma(z+b)$ при $z \rightarrow \infty$ (см. [17; с. 20]) и леммы 1.3 (подробнее см. [4]).

ОПредЕЛЕНиЕ 1.3. Через $C^{\lambda}\left(S_{n-1}\right), \operatorname{Re} \lambda>0$ обозначаем замыкание пространства $C^{\infty}\left(S_{n-1}\right)$ по норме $\|f\|_{C\left(S_{n-1}\right)}+\left\|D^{\lambda} f\right\|_{C\left(S_{n-1}\right)}$, где $D^{\lambda}$ произвольный оператор сферического дифференцирования (сферический оператор свертки) с мультипликатором класса $W_{\lambda, N}, N \geqslant[(n+1) / 2]$ ([· ] - целая часть числа).

ОПРЕДЕЛЕНИЕ 1.4. Пространствами переменной гёлъдеровости старших порядков на сфере $S_{n-1}$ назовем пространство

$$
H_{k}^{\lambda(x)}\left(S_{n-1}\right)=\left\{f: f(\sigma) \in C^{k}\left(S_{n-1}\right) ; A_{D^{k} f}<\infty\right\}, \quad k=1,2, \ldots,
$$

где $D^{k}$ - сферический оператор дифференцирования с мультипликатором из $W_{k, N}$, $N>n+1$. 
При таких условиях пространство $C^{\lambda}\left(H_{k}^{\lambda(x)}\right)$ с точностью до эквивалентности норм не зависит от выбора оператора $D^{\lambda}\left(D^{k}\right)$.

Доказательство первого факта приводится в [3], [12], при этом используется лемма 1.4 (представление (1.15)), а доказательство второго проводится по аналогии со случаем обычных и обобщенных гёльдеровских пространств ср. с [3], [5], [6].

Через $H_{0}^{\lambda(x)}\left(S_{n-1}\right)$ естественно обозначить $H^{\lambda(x)}\left(S_{n-1}\right)$.

Приведем несколько примеров таких функций.

Пример 1. $f(x)=\prod_{k=1}^{n}\left|x-x^{(k)}\right|^{\lambda_{k}}, 0<\lambda_{k} \leqslant 1, x, x^{(k)} \in S_{n-1}$. Тогда $f(x) \in$ $H^{\lambda(x)}\left(S_{n-1}\right)$, где $\lambda(x)=\lambda_{k}$ для $\left|x^{(i)}-x^{(j)}\right| \leqslant \varepsilon, \varepsilon=\frac{1}{4} \inf _{i \neq j}\left|x^{(i)}-x^{(j)}\right|$, и $\lambda(x)=1$ для всех других $x$.

Пример 2. Пусть $x \in S_{n-1}, f_{j}(x)=\left|x_{j}\right|+\sum_{k=2}^{\infty} 2^{-k} \sqrt{\left|x_{j}-k^{-1}\right|}, j=1,2, \ldots, n$. Понятно, что $f_{j}(x) \in H^{\lambda(x)}\left(S_{n-1}\right)$, где $\lambda(x)=2^{-1}$ при всех $x \in S_{n-1}, x_{j} \neq 0$ и $\lambda(x)=1, x \in S_{n-1}, x_{j}=0$.

ПримеР 3. Пусть $x \in S_{n-1}, Q=\left\{x_{j}^{k}\right\}_{k=1}^{\infty}$, где $k$ - верхний индекс, - упорядоченная последовательность рациональных чисел из $[-1,1]$. Представим $Q=\bigcup_{i} Q_{i}$, где $\left\{x_{j}^{k_{i}}\right\} \in Q_{i}$ и $1 /(k+1)<\left|x_{j}^{k_{i}}\right|<1 / k$. Положим

$$
\begin{gathered}
f_{j}(x)=\left|x_{j}\right|+\sum_{k=2}^{\infty} 2^{-k} \sum_{i=1}^{\infty}\left|x_{j}^{k_{i}}-x_{j}\right|^{\delta_{k_{i}}} 2^{-i}, \quad \delta_{k_{i}} \in(0,1), \\
\inf _{k, i} \delta_{k_{i}}=\delta_{0}>0, \quad j=1,2, \ldots, n .
\end{gathered}
$$

Тогда, очевидно, $f_{j}(x) \in H^{\lambda(x)}\left(S_{n-1}\right)$, где $\lambda(x)=\delta_{0}$ при всех $x \in S_{n-1}, x_{j} \neq 0$ и $\lambda(x)=1, x \in S_{n-1}, x_{j}=0$.

Различные варианты пространств такого типа ранее рассматривались в одномерном случае в работах [11], [13], [14], [18]-[20].

2. Сферические операторы в пространстве $H_{k}^{\lambda(x)}\left(S_{n-1}\right)$. Изоморфизм пространств переменной гёльдеровости. $1^{0}$. Случай $0<\operatorname{Re} \alpha<1$.

Teopema 2.1. Пyсmъ $f \in H^{\lambda(x)}\left(S_{n-1}\right) u \lambda_{+}>0, \operatorname{Re} \alpha>0, \lambda_{+}+\operatorname{Re} \alpha<1$. Тогдa $K^{\alpha} f \in H^{\lambda(x)+\operatorname{Re} \alpha}\left(S_{n-1}\right)$.

ДокАЗАТЕльство. Следуя идее доказательства из [5], [6], вначале укажем важное представление для разности $\left(K^{\alpha} f\right)(x)-\left(K^{\alpha} f\right)(y)$. Ясно, что нормировочный множитель $\gamma_{n-1}^{-1}(\alpha)$ войдет мультипликативно и в константу $c$ в правых частях последующих неравенств. По этой причине при доказательстве достаточно рассматривать оператор $K^{\alpha} f$ без него, что мы и будем делать. Кроме того, всюду в дальнейшем будем обозначать

$$
|x-y|=h
$$

и считать, что $0<h<1$. С учетом того, что в силу (1.1) и (1.5)

$$
\int_{S_{n-1}} \frac{d \sigma}{|x-\sigma|^{n-1-\alpha}}=\int_{S_{n-1}} \frac{d \sigma}{|y-\sigma|^{n-1-\alpha}},
$$


имеем

$$
\begin{aligned}
\left(K^{\alpha} f\right)(x)-\left(K^{\alpha} f\right)(y)= & \int_{|x-\sigma|<2 h} \frac{f(\sigma)-f(x)}{|x-\sigma|^{n-1-\alpha}} d \sigma-\int_{|x-\sigma|<2 h} \frac{f(\sigma)-f(x)}{|y-\sigma|^{n-1-\alpha}} d \sigma \\
& \quad+\int_{|x-\sigma|>2 h}[f(\sigma)-f(x)]\left\{|x-\sigma|^{\alpha-n+1}-|y-\sigma|^{\alpha-n+1}\right\} d \sigma \\
= & I_{1}+I_{2}+I_{3} .
\end{aligned}
$$

Слагаемые $I_{1}, I_{2}, I_{3}$ в (2.2) оцениваются по отдельности. Для $I_{1}$ имеем

$$
\begin{aligned}
\left|I_{1}\right| & \leqslant c \int_{|x-\sigma|<2 h} \frac{|\sigma-x|^{\lambda(x)}}{|x-\sigma|^{n-1-\operatorname{Re} \alpha}} d \sigma \leqslant c \int_{0}^{2 h} t^{\lambda(x)+\operatorname{Re} \alpha-1} d t \\
& \leqslant c \frac{h^{\lambda(x)+\operatorname{Re} \alpha}}{\lambda(x)+\operatorname{Re} \alpha} \leqslant c h^{\lambda(x)+\operatorname{Re} \alpha} .
\end{aligned}
$$

При оценке $I_{2}$ имеем

$$
\left|I_{2}\right| \leqslant c \int_{|y-\sigma|<2 h} \frac{|\sigma-x|^{\lambda(x)}}{|\sigma-y|^{n-1-\operatorname{Re} \alpha}} d \sigma .
$$

Применяя в (2.3) неравенство треугольника $|\sigma-x| \leqslant|y-\sigma|+|x-y|$, легко получаем

$$
\left|I_{2}\right| \leqslant c \int_{|y-\sigma|<3 h} \frac{|\sigma-y|^{\lambda(x)}}{|\sigma-y|^{n-1-\operatorname{Re} \alpha}} d \sigma+c h^{\lambda(x)+\operatorname{Re} \alpha} \int_{|y-\sigma|<3 h} \frac{d \sigma}{|\sigma-y|^{n-1-\operatorname{Re} \alpha}} .
$$

Здесь мы учли тот факт, что $\{\sigma:|x-\sigma|<2 h\} \subset\{\sigma:|\sigma-y|<3 h\}$. Окончательную оценку для $I_{2}$ можно получить, применяя (1.4) и вычисляя соответствующие интегралы:

$$
\left|I_{2}\right| \leqslant \operatorname{ch}^{\lambda(x)+\operatorname{Re} \alpha} \text {. }
$$

При оценке $I_{3}$, используя (1.12) при $\gamma=n-1-\alpha$, так что $\operatorname{Re} \gamma>0$, находим

$$
\left|I_{3}\right| \leqslant c h \int_{|x-\sigma|>2 h} \frac{|x-\sigma|^{\lambda(x)}}{|x-\sigma|^{n-\operatorname{Re} \alpha}} d \sigma \leqslant c h \int_{2 h}^{2} \frac{u^{\lambda(x)}}{u^{2-\operatorname{Re} \alpha}} d u .
$$

Далее, вычисляя в (2.4) интеграл, получаем

$$
\left|I_{3}\right| \leqslant c \frac{h^{\lambda(x)+\operatorname{Re} \alpha}}{1-\lambda(x)-\operatorname{Re} \alpha} .
$$

Так как $\lambda_{+}+\operatorname{Re} \alpha<1$, из (2.5) следует

$$
\left|I_{3}\right| \leqslant \operatorname{ch}^{\lambda(x)+\operatorname{Re} \alpha} .
$$

Что и требовалось доказать.

ЗАмЕчАниЕ 2.1. После анализа доказательства теоремы 2.1 становится понятным и случай $\lambda_{+}+\operatorname{Re} \alpha=1$. Здесь, очевидно,

$$
\left|\left(K^{\alpha} f\right)(x)-\left(K^{\alpha} f\right)(y)\right| \leqslant \frac{c h^{\lambda(x)+\operatorname{Re} \alpha}}{1-\lambda(x)-\operatorname{Re} \alpha}
$$

для всех $y \in S_{n-1}$ и тех $x \in S_{n-1}$, для которых $\lambda(x)+\operatorname{Re} \alpha<1$ и

$$
\left|\left(K^{\alpha} f\right)(x)-\left(K^{\alpha} f\right)(y)\right| \leqslant c h^{\lambda(x)+\operatorname{Re} \alpha} \ln h^{-1}
$$

для всех $x, y \in S_{n-1}$, где $\lambda(x)+\operatorname{Re} \alpha=1$. 
Teopema 2.2. Пyсmъ $f \in H^{\lambda(x)}\left(S_{n-1}\right), \lambda_{-}>0, \operatorname{Re} \alpha>0,0<\lambda_{-}-\operatorname{Re} \alpha<1 u$ выполнено условие (0.9). Тогда $D^{\alpha} f \in H^{\lambda(x)-\operatorname{Re} \alpha}\left(S_{n-1}\right)$.

ДокАЗАТЕЛЬСтво. Как и выше, при доказательстве используем запись для $D^{\alpha} f$ без мультипликативной постоянной $\gamma_{n-1}^{-1}(-\alpha)$. Имеем

$$
\begin{aligned}
& \left(D^{\alpha} f\right)(x)-\left(D^{\alpha} f\right)(y) \\
& =\int_{S_{n-1}} \frac{f(\sigma)-f(x)}{|x-\sigma|^{n-1+\alpha}} d \sigma-\int_{S_{n-1}} \frac{f(\sigma)-f(y)}{|y-\sigma|^{n-1+\alpha}} d \sigma=A_{1}+A_{2}+A_{3}+A_{4} \\
& =\int_{|x-\sigma|<2 h} \frac{f(\sigma)-f(x)}{|x-\sigma|^{n-1+\alpha}} d \sigma-\int_{|x-\sigma|<2 h} \frac{f(\sigma)-f(y)}{|y-\sigma|^{n-1+\alpha}} d \sigma \\
& \quad+[f(x)-f(y)] \int_{|x-\sigma|>2 h} \frac{d \sigma}{|\sigma-y|^{n-1+\alpha}} \\
& \quad+\int_{|x-\sigma|>2 h}[f(\sigma)-f(x)]\left\{|x-\sigma|^{-\alpha-n+1}-|y-\sigma|^{-\alpha-n+1}\right\} d \sigma .
\end{aligned}
$$

Для $A_{1}$ имеем

$$
\left|A_{1}\right| \leqslant c \int_{|x-\sigma|<2 h} \frac{|\sigma-x|^{\lambda(x)}}{|x-\sigma|^{n-1+\operatorname{Re} \alpha}} d \sigma \leqslant c \int_{0}^{2 h} t^{\lambda(x)-\operatorname{Re} \alpha-1} d t \leqslant c \frac{h^{\lambda(x)-\operatorname{Re} \alpha}}{\lambda(x)-\operatorname{Re} \alpha} .
$$

Так как $\lambda_{-}-\operatorname{Re} \alpha>0$, то

$$
\left|A_{1}\right| \leqslant c h^{\lambda(x)-\operatorname{Re} \alpha}
$$

При оценке $A_{2}$ имеем

$$
\left|A_{2}\right| \leqslant c \int_{|y-\sigma|<3 h} \frac{|\sigma-y|^{\lambda(y)}}{|\sigma-y|^{n-1+\operatorname{Re} \alpha}} d \sigma \leqslant c h^{\lambda(y)-\operatorname{Re} \alpha} .
$$

Здесь мы учли оценку (2.7) и тот факт, что $\{\sigma:|x-\sigma|<2 h\} \subset\{\sigma:|\sigma-y|<3 h\}$. Далее, при $\lambda(y)-\lambda(x) \geqslant 0$ очевидно, что

$$
\left|A_{2}\right| \leqslant c h^{\lambda(x)-\operatorname{Re} \alpha} .
$$

При $\lambda(y)-\lambda(x)<0$ с учетом (0.9) получаем

$$
\left|A_{2}\right| \leqslant c h^{\lambda(x)-\operatorname{Re} \alpha} \exp \left(|\lambda(y)-\lambda(x)||\ln h|^{-1}\right) \leqslant c h^{\lambda(x)-\operatorname{Re} \alpha} .
$$

Ввиду оценки (1.6) имеем

$$
\left|A_{3}\right| \leqslant c h^{\lambda(x)-\operatorname{Re} \alpha} .
$$

При оценке $A_{4}$, используя (1.12) при $\gamma=n-1+\alpha$, так что $\operatorname{Re} \gamma>0$, получаем

$$
\left|A_{4}\right| \leqslant \operatorname{ch} \int_{|x-\sigma|>2 h} \frac{|x-\sigma|^{\lambda(x)}}{|x-\sigma|^{n+\operatorname{Re} \alpha}} d \sigma \leqslant c h \int_{2 h}^{2} \frac{u^{\lambda(x)}}{u^{2+\operatorname{Re} \alpha}} d u .
$$

Далее, вычисляя интеграл в (2.10), получаем

$$
\left|A_{4}\right| \leqslant c \frac{h^{\lambda(x)-\operatorname{Re} \alpha}}{1-\lambda(x)+\operatorname{Re} \alpha} \leqslant c h^{\lambda(x)-\operatorname{Re} \alpha} .
$$

Собирая полученные оценки, получаем нужное. 
$2^{0}$. Случай $\operatorname{Re} \alpha=0, \alpha \neq 0$. Рассмотрим теперь оператор $D^{\alpha}$ в случае, когда $\alpha= \pm i \theta$ чисто мнимое, понимая его, как и при $\operatorname{Re} \alpha>0$, в виде $(0.2)$. Оператор $D^{ \pm i \theta}$ обладает как свойствами сферического потенциала, так и гиперсингулярного интеграла.

Для дальнейшего нам понадобится следующая вспомогательная

Лемма 2.1. Пусть $\theta \in \mathbb{R}^{1} \backslash\{0\}$. Тогда $\sup _{0<h<1}|I(h)|<\infty$, где

$$
I(h)=\int_{|x-\sigma|>2 h} \frac{d \sigma}{|y-\sigma|^{n-1+i \theta}}, \quad h=|x-y| .
$$

ДокАЗАТЕЛЬСтво. Заметим, что интеграл в (2.12) инвариантен относительно всех вращений по $x$ и $y$ на сфере и, следовательно, зависит только от $h=|x-y|$, так что обозначение $I(h)$ корректно. Используя аддитивные свойства интеграла, имеем

$$
I(h)=\int_{\substack{|x-\sigma|>2 h \\|y-\sigma|>2 h}} \frac{d \sigma}{|y-\sigma|^{n-1+i \theta}}+\int_{\substack{|x-\sigma|>2 h \\ h<|y-\sigma|<2 h}} \frac{d \sigma}{|y-\sigma|^{n-1+i \theta}}=\widetilde{I}_{1}+\widetilde{I}_{2} .
$$

Оценка $\widetilde{I}_{2}$ проста, поскольку с учетом (1.4) имеем

$$
\left|\widetilde{I}_{2}\right| \leqslant c \int_{h}^{2 h} \frac{d u}{u}=c \ln 2 .
$$

Для оценки $\widetilde{I}_{1}$ представим его в следующем виде:

$$
\left|\widetilde{I}_{1}\right|=\int_{|y-\sigma|>2 h} \frac{d \sigma}{|y-\sigma|^{n-1+i \theta}}-\int_{\substack{h<|x-\sigma|<2 h \\|y-\sigma|>2 h}} \frac{d \sigma}{|y-\sigma|^{n-1+i \theta}}=\widetilde{I}_{1}^{\prime}+\widetilde{I}_{1}^{\prime \prime} .
$$

Интеграл $\widetilde{I}_{1}^{\prime \prime}$ также оценивается абсолютно и подобно $\widetilde{I}_{2}$, так что $\left|\widetilde{I}_{1}^{\prime \prime}\right| \leqslant c \ln 2$. Итак, остается оценить $\widetilde{I}_{1}^{\prime}$. На основе (1.3) имеем

$$
\widetilde{I}_{1}^{\prime}=2^{3-n} C_{n} \int_{2 h}^{2} u^{-1+i \theta}\left[\left(4-u^{2}\right)^{(n-3) / 2}-2^{n-3}\right] d u+C_{n} \int_{2 h}^{2} u^{-1+i \theta} d u=\widetilde{\widetilde{I}}_{1}^{\prime}+\widetilde{\widetilde{I}}_{1}^{\prime \prime} .
$$

Интеграл $\widetilde{\widetilde{I}}_{1}^{\prime}$ сходится абсолютно $(n \geqslant 2)$. Что касается $\widetilde{\widetilde{I}}_{1}^{\prime \prime}$, то непосредственная оценка дает $\left|\widetilde{\widetilde{I}}_{1}^{\prime \prime}(h)\right| \leqslant 2 C_{n} /|\theta|$. Лемма доказана.

Tеорема 2.3. Пусть $f \in H^{\lambda(x)}\left(S_{n-1}\right)$ и выполнено условие (0.9). Тогда $\mathscr{D}^{ \pm i \theta} f \in$ $H^{\lambda(x)}\left(S_{n-1}\right)$.

ДоКАЗАТЕЛЬСтво. Мы можем использовать представление (2.6) и при $\alpha= \pm i \theta$, а далее нужно только проанализировать, где следует видоизменить доказательство. Ясно, что оценки $\left|A_{1}\right|$ и $\left|A_{2}\right|$ сохраняются. При оценке $\left|A_{4}\right|$ мы приходим к оценкам типа $(2.10),(2.11)$ и на этом останавливаемся. Наконец, оценивая $A_{3}$, например, для случая $\alpha=i \theta$, имеем $\left|A_{3}\right| \leqslant h^{\lambda(x)}|I(h)|$ и остается воспользоваться леммой 2.1, что приводит к оценке $\left|A_{3}\right| \leqslant c h^{\lambda(x)}$. Объединяя все оценки, получаем требуемое.

$3^{0}$. Изоморфизм пространств переменной гёльдеровости.

Tеорема 2.4. Пусть $f \in H_{k}^{\lambda(x)}\left(S_{n-1}\right)$, Re $\alpha>0, \alpha \neq n-1, n+1, \ldots, \lambda_{+}+$ $\{\operatorname{Re} \alpha\}<1$ и выполнено условие $(0.9)$. Тогда $K^{\alpha}$ осуществляет изоморбизм между пространствами $H_{k}^{\lambda(x)}\left(S_{n-1}\right)$ u $H_{k+[\operatorname{Re} \alpha]}^{\lambda(x)+\{\operatorname{Re} \alpha\}}\left(S_{n-1}\right)$, a оператор $\mathscr{D}^{ \pm i \theta}$ сохраняет пространство $H_{k}^{\lambda(x)}\left(S_{n-1}\right)$, т.е. справедливы представления (0.7) и (0.8). 
Доказательство. Пусть $0<\operatorname{Re} \alpha<1, k=1$. Первое утверждение теоремы 2.4 следует из теорем 2.1, 2.2, представления (0.5) и вложений $H^{\lambda_{+}}\left(S_{n-1}\right) \subset$ $H^{\lambda(x)}\left(S_{n-1}\right) \subset H^{\lambda_{-}}\left(S_{n-1}\right)$, а второе из теоремы 2.3 и представления $(0.6)$.

Случай $\operatorname{Re} \alpha \geqslant 1, k \geqslant 1$ сводится к случаю $0<\operatorname{Re} \alpha<1, k=0$ за счет выбора $D^{[\operatorname{Re} \alpha]}: D^{[\operatorname{Re} \alpha]} K^{\alpha}=K^{\alpha-[\operatorname{Re} \alpha]}$.

Теорема 2.5. Пусть оператор $A^{\alpha}$ имеет мультипликатор $\left\{k_{m}\right\}_{m=0}^{\infty}$. Eсли $\left\{k_{m}\right\}_{m=0}^{\infty} \in W_{-\alpha, N}, \operatorname{Re} \alpha>0, N>n+1, k_{m} \neq 0, m=0,1, \ldots, \alpha \neq n-1, n+1, \ldots$, mo

$$
A^{\alpha}\left(H_{k}^{\lambda(x)}\left(S_{n-1}\right)\right)=H_{k+[\operatorname{Re} \alpha]}^{\lambda(x)+\{\operatorname{Re} \alpha\}}\left(S_{n-1}\right)
$$

при выполнении условия (0.9).

ДокАЗАтЕЛьство. Здесь также достаточно рассмотреть случай $k=0$ и $0<$ $\operatorname{Re} \alpha<1$, так как случай $\operatorname{Re} \alpha \geqslant 1$ получается за счет выбора $D^{[\operatorname{Re} \alpha]}: D^{[\operatorname{Re} \alpha]} A^{\alpha}=$ $A^{\alpha-[\operatorname{Re} \alpha]}$. Для оператора $A^{\alpha}$ в силу (1.16) из леммы 1.4 справедливо представление

$$
A^{\alpha}=K^{\alpha}(c I+B) .
$$

Так как оператор $B$ имеет структуру

$$
B=\int_{S_{n-1}} k(x \cdot \sigma) \varphi(\sigma) d \sigma+\sum_{j=1}^{N} c_{j} K^{j}
$$

где $k(t) \in C^{1}([-1,1])$, а $K^{j}$ - потенциалы целого порядка $j$, очевидно, он сохраняет переменную гёльдеровость и поэтому в силу теоремы 2.4 и изоморфизма (0.7) мы получаем нужное.

ЗАмЕчАниЕ 2.2. Утверждение теоремы 2.5 означает существование и единственность решения многомерного интегрального уравнения первого рода, порождаемого оператором (1.14), в пространствах гёльдеровских функций переменного порядка.

К операторам, удовлетворяющим условиям теоремы 2.5 , относится ряд известных операторов сферической свертки, см., например, [2]-[4], [12]:

$$
\begin{array}{ll}
\left(K_{1}^{\alpha} f\right)(x)=\frac{1}{\Gamma(\alpha)} \int_{0}^{1}(1-r)^{\alpha-1} P_{r} f(t) d r, & \operatorname{Re} \alpha>0, \\
\left(K_{2}^{\alpha} f\right)(x)=\frac{1}{\Gamma(\alpha)} \int_{0}^{1}\left(\ln \frac{1}{r}\right)^{\alpha-1} P_{r} f(t) d r, & \operatorname{Re} \alpha>0,
\end{array}
$$

где

$$
P_{r} f(x)=\frac{1}{c_{n}} \int_{S_{n-1}} \frac{\left(1-r^{2}\right)}{\left|r x^{\prime}-\sigma\right|^{n}} f(\sigma) d \sigma, \quad x^{\prime}=\frac{x}{|x|}, \quad r=|x|, \quad x \in \mathbb{R}^{n},
$$

a $c_{n}$ - хорошо известная нормировочная постоянная для оператора Пуассона,

$$
\left(K_{3}^{\alpha} f\right)(x)=\int_{S_{n-1}} \chi_{n-\alpha}\left(\frac{1+x \cdot \sigma}{2}\right) f(\sigma) d \sigma
$$

где

$$
\chi(z)=\sum_{j=2}^{\infty} \frac{(\beta)_{j} z^{j}}{((\beta+1) / 2)_{j} j}, \quad 0 \leqslant z \leqslant 1, \quad(\beta)_{j}=\beta(\beta+1) \cdots(\beta+j-1),
$$


с мультипликаторами по сферическим гармоникам

$$
k_{m}^{1}=\frac{1}{(m+1)^{\alpha}}, \quad k_{m}^{2}=\frac{\Gamma(m+1)}{\Gamma(m+1+\alpha)}, \quad k_{m}^{3}=\frac{\Gamma((m+n-\alpha) / 2) \Gamma(m / 2)}{\Gamma((m+\alpha) / 2) \Gamma((m+n) / 2)}
$$

соответственно.

$4^{0}$. Операторы типа потенциала с особенностями на полюсах в пространствах гёльдеровских функций переменного порядка. Рассмотрим двуполюсный потенциал

$$
\left(\mathbf{K}^{\alpha} f\right)(x)=c \int_{S_{n-1}} \frac{f(\sigma)}{|x-\sigma|^{n-1-\alpha}|x+\sigma|^{n-1-\alpha}} d \sigma, \quad x \in S_{n-1},
$$

где $n \geqslant 3, \operatorname{Re} \alpha>0$ и $c$ - некоторая нормировочная постоянная,имеющий мультипликатор по сферическим гармоникам (см. [21; формула (18) с. 519]):

$$
k_{m}=c 2^{\alpha-n+1}\left|S_{n-2}\right|(-1)^{m} \sqrt{\pi} \frac{\Gamma(\alpha / 2)}{\Gamma((\alpha+1) / 2)}{ }_{3} F_{2}\left(-m, m+n-2, \frac{\alpha}{2}, \alpha, \frac{n-1}{2} ; 1\right) \text {. }
$$

Используя формулу (4), с. 173 из [17], после несложных преобразований, из (2.13) получаем

$$
k_{m}=c_{1}\left[1+(-1)^{m}\right] \frac{\Gamma((m+1) / 2) \Gamma((m-\alpha+n-1) / 2)}{\Gamma((m+n-1) / 2) \Gamma((1+m+\alpha) / 2)} \sim c m^{-\alpha}, \quad m \rightarrow \infty,
$$

где

$$
c_{1}=c 2^{\alpha-n}\left|S_{n-2}\right| \pi^{-1} \Gamma\left(\frac{n-1}{2}\right) \Gamma\left(\frac{\alpha}{2}\right) \Gamma\left(\frac{\alpha+3-n}{2}\right) \sin \frac{(n-\alpha-1) \pi}{2} .
$$

Обозначим $A=\frac{1}{2}(I+Q)$, где $Q$ оператор отражения на сфере, действующий по правилу $(Q f)(x)=f(-x)$. Ясно, что оператор $A$ имеет мультипликатор вида $k_{m}=0$, если $m$ нечетное и $k_{m}=1$, если $m$ четное.

Лемма 2.2. Пусть $\operatorname{Re} \alpha>0, \alpha \neq n-1, n+1, \ldots$. Тогда

$$
\mathbf{K}^{\alpha}=A\left(c_{2} I+B_{1}\right) K^{\alpha},
$$

где $c_{2}$ - некоторая константа, порождаемая асимптотическим разложением мультипликатора оператора $K^{\alpha}$, а оператор $B_{1}$ сохраняет переменную гёлъдеровость.

ДокАзАтельство. Доказательство (2.15) вытекает из представления (2.14) для мультипликатора $\mathbf{K}^{\alpha}$, известного асимптотического представления для $\Gamma(z+a) /$ $\Gamma(z+b)$ (см. [17; с. 20] и леммы 1.4; подробнее см. также [4]).

Tеорема 2.6. Пусть $f \in H_{k}^{\lambda(x)}\left(S_{n-1}\right), \operatorname{Re} \alpha>0, \lambda_{+}+\{\operatorname{Re} \alpha\}<1$. Тогда справедливо вложение

$$
\mathbf{K}^{\alpha}\left(H_{k}^{\lambda(x)}\left(S_{n-1}\right)\right) \rightarrow H_{k+[\operatorname{Re} \alpha]}^{\lambda(x)+\{\operatorname{Re} \alpha\}}\left(S_{n-1}\right) .
$$

ДокАЗАтЕЛЬСтво. Утверждение теоремы следует из представления (2.14), теоремы 2.4 и того факта, что операторы $A$ и $B_{1}$ сохраняют переменную гёльдеровость.

ЗАмечаниЕ 2.3. Очевидно, что оператор $\mathbf{K}^{\alpha}$ не удовлетворяет одному из условий теоремы 2.5 , а именно тому, что $k_{m} \neq 0, m=0,1, \ldots$, поэтому вместо изоморфизма мы имеем одностороннее вложение (2.16). Однако если рассматривать только четные функции, то для них соответствующий изоморфизм имеет место при условии (0.9). 


\section{3. Риссов потенциал по $\dot{\mathbb{R}}^{n}$ в пространствах переменной гёльдеровости} с некоторыми степенными весами. Рассмотрим риссов потенциал по $\mathbb{R}^{n}$

$$
\left(K^{\alpha} f\right)(\xi)=\frac{1}{\gamma_{n}(\alpha)} \int_{\mathbb{R}^{n}} \frac{f(\eta)}{|\xi-\eta|^{n-\alpha}} d \eta, \quad \xi \in \mathbb{R}^{n}, \quad 0<\operatorname{Re} \alpha<n,
$$

в пространствах переменной гёльдеровости в $\mathbb{R}^{n}$ с весом $H^{\lambda(\xi)}\left(\dot{\mathbb{R}}^{n}, r\right)=\{f: r f \in$ $\left.H^{\lambda(\xi)}\left(\dot{\mathbb{R}}^{n}\right)\right\}$, где

$$
\begin{gathered}
H^{\lambda(\xi)}\left(\dot{\mathbb{R}}^{n}\right)=\left\{f: f \in C\left(\dot{\mathbb{R}}^{n}\right) ; \sup _{\eta, \xi \in \mathbb{R}^{n}} \frac{|f(\eta)-f(\xi)|}{\left(|\eta-\xi| / \sqrt{\left(1+|\eta|^{2}\right)\left(1+|\xi|^{2}\right)}\right)^{\lambda(\xi)}}<\infty\right\}, \\
0<\lambda_{-} \leqslant \lambda(\xi) \leqslant \lambda_{+}<1, \quad \lambda_{-}=\inf _{\xi \in \mathbb{R}^{n}} \lambda(\xi), \quad \lambda_{+}=\sup _{\xi \in \mathbb{R}^{n}} \lambda(\xi) .
\end{gathered}
$$

Teоpema 3.1. Onератор (3.1), $0<\operatorname{Re} \alpha, \lambda_{+}+\operatorname{Re} \alpha<1$, изоморфно отображает $H^{\lambda(\xi)}\left(\dot{\mathbb{R}}^{n},(1+|\xi|)^{(n+\alpha) / 2}\right)$ на $H^{\lambda(\xi)+\operatorname{Re} \alpha}\left(\dot{\mathbb{R}}^{n},(1+|\xi|)^{(n-\alpha) / 2}\right)$ nри условии, что $\widetilde{\lambda}(x)=$ $\lambda\left(x_{1} /\left(1-x_{n+1}\right), \ldots, x_{n} /\left(1-x_{n+1}\right)\right)$ удовлетворяет условию (0.9) для всех $x \in S_{n}$, $S_{n} \subset \mathbb{R}^{n+1}$.

ДокАЗАТЕЛЬСтво. Утверждение теоремы следует из теоремы 2.4 с помощью стереографической проекции сферы $S_{n} \subset \mathbb{R}^{n+1}$ на $\dot{\mathbb{R}}^{n}$ (случай одноточечной компактификации $\left.\mathbb{R}^{n}\right)$, которая переводит оператор $(0.1)$ по $S_{n}$ в риссов потенциал (с весом $\left.(1+|\xi|)^{(n-\alpha) / 2}\right)$ по $\mathbb{R}^{n}$. Действительно, известно, что вектор-функция преобразования координат $v(\xi)$ при этом преобразовании имеет вид (см. [22; с. 35])

$$
\sigma_{k}=\frac{\xi_{k}}{1+|\xi|^{2}}, \quad k=0,1, \ldots, n, \quad \sigma_{n+1}=\frac{1-|\xi|^{2}}{1+|\xi|^{2}}
$$

а также справедливы следующие формулы:

$$
|x-\sigma|=\frac{|\xi-\eta|}{\sqrt{\left(1+|\xi|^{2}\right)\left(1+|\eta|^{2}\right)}}, \quad d \sigma=\frac{2^{n} d \xi}{\left(1+|\xi|^{2}\right)^{n}},
$$

где $\xi, \eta \in \dot{\mathbb{R}}^{n}$, а $x, \sigma \in S_{n}$ - их образы при стереографической проекции. Поэтому с учетом (3.1), (3.2) имеем

$$
\int_{S_{n}} \frac{f(\sigma)}{|x-\sigma|^{n-\alpha}} d \sigma=2^{\alpha}\left(1+|\eta|^{2}\right)^{(n-\alpha) / 2} \int_{\mathbb{R}^{n}} \frac{f(v(\eta))\left(1+|\xi|^{2}\right)^{(-n-\alpha) / 2}}{|\xi-\eta|^{n-\alpha}} d \eta .
$$

Кроме того, очевидно, что при стереографической проекции класс $H^{\lambda(x)}\left(S_{n-1}\right)$ переходит в класс $H^{\lambda(v(\xi))}\left(\dot{\mathbb{R}}^{n}\right)$. Отсюда и следует требуемое.

Утверждение типа теоремы 3.1 было известно ранее для обобщенных пространств Гёльдера с характеристиками из классов типа Бари-Стечкина (см. [5]).

\section{СПИСОК ЦИТИРОВАННОЙ ЛИТЕРАТУРЫ}

[1] П. М. Павлов, С. Г. Самко, "Описание пространств $L_{p}^{\alpha}\left(S_{n-1}\right)$ в терминах гиперсингулярных интегралов", Докл. АН СССР, 276:3 (1984), 546-550.

[2] С. Г. Самко, "Сингулярные интегралы по сфере и построение характеристики по символу", Изв. вузов. Матем., 1983, № 4, 28-42. 
[3] S. G. Samko, B. G. Vakulov, "On equivalent norms in fractional order functions spaces of continuous functions on the unit sphere", Fract. Calc. Appl. Anal., 3:4 (2000), 401-433.

[4] S. G. Samko, "Hypersingular integrals and their applications", Analytical Methods and Special Functions, 5, Taylor \& Francis, London-New York, 2002, 358-373.

[5] Б. Г. Вакулов, "Операторы типа потенциала на сфере в обобщенных классах Гёльдера", Изв. вузов. Матем., 1986, № 11, 66-69.

[6] Б. Г. Вакулов, "Операторы типа потенциала на сфере в обобщенных пространствах Гёльдера”, Деп. ВИНИТИ № 1563-B86 (06.05.1986), Ростовский ун-т, Ростов-на-Дону, 1986.

[7] Б. Г. Вакулов, "Сферические операторы типа потенциала в обобщенных пространствах Гёльдера с весом на сфере", Изв. вузов Сев.-Кавк. региона. Естеств. науки, 1999, № 4, 5-10.

[8] Б. Г. Вакулов, Н. К. Карапетянц, Л. Д. Шанкишвили, "Сферические потенциалы комплексного порядка в обобщенных пространствах Гёльдера с весом”, Докл. РАН, 382:3 (2002), 1-4.

[9] B. G. Vakulov, N. K. Karapetians, L. D. Shankishvili, "Spherical hypersingular operators of imaginary order and their multipliers", Fract. Calc. Appl. Anal., 4:1 (2001), 101-112.

[10] Б. Г. Вакулов, Н. К. Карапетянц, Л. Д. Шанкишвили, "Сферические потенциалы комплексного порядка в обобщенных гёльдеровых пространствах", Изв. НАН Армении, 36:2 (2001), 54-78.

[11] А. И. Гинзбург, Н. К. Карапетянц, “Дробное интегродифференцирование в гёльдеровских классах переменного порядка”, Докл. РАН, 339:4 (1994), 439-441.

[12] Б. Г. Вакулов, “Об эквивалентных нормировках в пространствах функций комплексной гладкости на сфере", Тр. ИМ НАН Беларуси, 9, Минск, 2001, 41-44.

[13] N. K. Karapetians, A. I. Ginzburg, "Fractional integrodifferentiation in Hölder classes of arbitrary order", Georgian Math. J., 2:2 (1995), 141-150.

[14] N. K. Karapetians, A. I. Ginzburg, "Fractional integrals and singular integrals in the Hölder classes of variable order", Integral Transform. Spec. Funct., 2:2 (1994), 91-106.

[15] B. Ross, S. Samko, "Fractional integration operator of variable order in the Hölder spaces

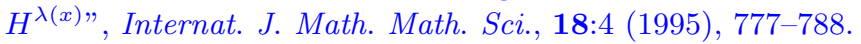

[16] С.Г. Самко, Гиперсингулярные интегралы и их приложения, Изд-во РГУ, Ростовна-Дону, 1984.

[17] А.П. Прудников, Ю.А. Брычков, О.И. Маричев, Интегралы и рлды. Специальные функции, Наука, М., 1983.

[18] K. Daodi, J. Levy Vehel, Y. Meyer, "Construction of continuous functions with prescribed local regularity", Constructive Approximation, 14:3 (1998), 349-385.

[19] Д.И. Мамедханов, А.А. Нерсесян, "О конструктивной характеристике класса $H_{\alpha}^{\lambda+\alpha}\left(x_{0},[-\pi, \pi]\right) "$, Исследования по теории линейных операторов, Баку, 1987, 74-78.

[20] Н. Б. Плещинский, "О построении функций, удовлетворяющих условию Гёльдера с заданным показателем", Изв. вузов. Матем., 1984, № 8, 74-77.

[21] Ю. Люк, Специальные математические функиии и их аппроксимации, Мир, М., 1980.

[22] С. Г. Михлин, Многомерные сингулярные интегралы и интегральные уравнения, Физматгиз, М., 1962.

\section{Б. Г. Вакулов}

Ростовский государственный университет

E-mail: vakulov@ns.math.rsu.ru
Поступило

07.08.2003 